\title{
Modest Improvement of Untreated Severe Sleep-Disordered Breathing in the Middle-Aged and Elderly
}

\author{
Hong Jun Jeon 1 , Young Rong Bang², Soyeon Jeon², \\ Tae Young Lee ${ }^{3}$, Hye Youn Park², and In-Young Yoon ${ }^{2,4} \bowtie$ \\ 'Department of Psychiatry, Konkuk University Medical Center, Seoul, Republic of Korea \\ ${ }^{2}$ Department of Psychiatry, Seoul National University Bundang Hospital, Seungnam, Republic of Korea \\ ${ }^{3}$ Medical Research Center, Seoul National University Hospital, Seoul, Republic of Korea \\ ${ }^{4}$ Department of Psychiatry, School of Medicine, Seoul National University, Seoul, Republic of Korea
}

Objective It has been reported that untreated sleep-disordered breathing (SDB) deteriorates over time, however this remains contentious. The aim of the present study is to evaluate the clinical course of SDB in middle-aged and older SDB patients, and to identify how relevant factors contribute to the change in SDB severity.

Methods Baseline and follow-up polysomnographic data of 56 untreated SDB patients (mean age, 61.2 \pm 5.71 ) were obtained retrospectively and the mean interval was $62.4 \pm 22.0$ months. Subgroup analysis was performed based on the baseline severity, and the factors associated with the course of SDB were analyzed.

Results At the baseline, 13 subjects were simple snorers, 15 had mild to moderate SDB, and 28 were severe SDB patients. While there was no significant change in apnea-hypopnea index (AHI) as a whole, subgroup analysis showed decrease of AHI in severe SDB patients (43.9 \pm 10.6 to $35.6 \pm 20.0, \mathrm{p}=0.009)$. The change in supine time percent and baseline AHI were associated with the change in $\mathrm{AHI}(\beta=0.387$, $\mathrm{p}=0.003 ; \beta=-0.272, \mathrm{p}=0.037)$.

Conclusion Untreated SDB did not deteriorate over time with modest improvement in severe SDB. A proportion of severe SDB patients might expect decrease in SDB severity irrespective of changes in sleep position or body weight.

Psychiatry Investig 2017;14(5):662-668

Key Words Apnea-hypopnea index, Clinical course, Sleep position, Sleep-disordered breathing.

\section{INTRODUCTION}

Sleep-disordered breathing (SDB) is a common disorder affecting 9.0 to $15.0 \%$ of middle-aged adults, ${ }^{1}$ and it is much more prevalent in elderly population, affecting 52.6 to $70.0 \%$ of men and 26.3 to $56.0 \%$ of women. ${ }^{2,3}$ SDB is characterized by pharyngeal obstruction during sleep which causes recurrent cessation of breathing, and is associated with a wide range of co-morbidities, including hypertension, metabolic syndrome and cardiovascular diseases. ${ }^{4-7}$ Long-term follow-up studies

Received: September 11, 2016 Revised: October 18, 2016

Accepted: November 19, 2016 Available online: July 26, 2017

$\triangle$ Correspondence: In-Young Yoon, MD, PhD

Department of Psychiatry, Seoul National University Bundang Hospital, 166 Gumi-ro, Bundang-gu, Seongnam 13620, Republic of Korea

Tel: +82-31-787-4058, Fax: +82-31-787-7433, E-mail: iyoon@snu.ac.kr

(c) This is an Open Access article distributed under the terms of the Creative Commons Attribution Non-Commercial License (http://creativecommons.org/licenses/by$\mathrm{nc} / 4.0$ ) which permits unrestricted non-commercial use, distribution, and reproduction in any medium, provided the original work is properly cited. on such co-morbidities of SDB are mostly based on the assumption that the severity at the time of diagnosis does not change over time. However, previous studies reported conflicting results on the clinical course of SDB. Whether SDB progresses or not remains an important issue among clinicians and health care professionals, because the information on SDB evolution may be required to determine the long-term morbidity and mortality of individuals with SDB.

The majority of previous studies on the clinical course of $\mathrm{SDB}$, either of general population ${ }^{8-11}$ or clinical samples, ${ }^{12-14}$ reported that untreated SDB has a tendency to deteriorate. However, there also have been considerable numbers of studies ${ }^{15-17}$ that reported conflicting results, showing from long-term stability of the disease to improvement of SDB, especially in severe cases.

Confounding factors are assumed to be related with controversies in results of previous studies on the clinical course of SDB. Most of changes in SDB severity shown by previous stud- 
ies were significantly associated with weight change. Only two studies have reported that changes in weight did not influence the progression of SDB. ${ }^{18,19}$ Caution also should be taken in interpreting studies on the clinical course of SDB due to variability in sleeping positions. ${ }^{12,15,20}$ Given the role of supine position during sleep on the severity of upper airway resistance in SDB individuals, ${ }^{21,22}$ the change in percentage of supine time between examinations is presumed to be considerably important in judging the evolution of SDB.

As such, the aim of the present study is to evaluate the clinical course of SDB in untreated middle-aged and older SDB patients, and to identify how relevant factors contribute to the change in SDB severity.

\section{METHODS}

\section{Study design and subjects}

This is a retrospective study, carried out at the sleep center of the Seoul National University Bundang Hospital. Patients who were diagnosed as SDB and did not receive treatment, were included if two overnight PSGs were conducted between 2004 and 2015. The patients went through two overnight PSGs in the process of recruitment for our previous study. ${ }^{23}$ The interval between the two PSGs was at least 24 months, or longer. Subjects were not treated in the case of mild severity, intolerable discomfort of oral appliances or continuous positive airway pressure (CPAP) therapy, or refusal of treatment for other reasons. As we aimed to study the clinical course of SDB in middle-aged and elderly population, subjects who were under 45 years of age were excluded. Demographic and anthropometric data were obtained, such as height, weight, body mass index (BMI), waist and hip circumference and blood pressure. Information on sleepiness was obtained from the Epworth Sleepiness Scale (ESS). This study was approved by the Institutional Review Board of Seoul National University Bundang Hospital. Informed consent was obtained from all individual participants included in the study.

\section{Polysomnography}

All subjects underwent laboratory overnight PSG (Embla $^{\mathrm{TM}}$ N7000, Embla, Reykjavik, Iceland) with standard electrodes and sensors. ${ }^{24}$ Electroencephalography electrodes were applied at O1/A2, O2/A1, C4/A1, and C3/A2. Two electrooculographies were applied at the sides of both eyes for recording vertical and horizontal eye movements. Electromyography electrodes were applied at the submentalis muscles and both anterior tibialis muscles. Strain gauges were applied at the chest and abdomen to record respiratory movements. Nasal pressure transducers were used to record airflow and pulse oximeters were applied on the index finger to measure the arterial oxygen saturation. The recordings were scored based on the standard criteria of Kales and Rechtscahffen. ${ }^{25}$

\section{SBD-related variables}

Apnea was defined as an episode of complete air flow cessation of at least $10 \mathrm{~s}$. Hypopnea was defined as $50 \%$ or more reduction of airflow for at least $10 \mathrm{~s}$, or moderate airflow reduction for at least $10 \mathrm{~s}$ accompanied by electroencephalographic arousal, or by oxygen desaturation $(\geq 4 \%)$. ${ }^{26}$ The severity of SDB was evaluated by apnea-hypopnea index (AHI), defined as the number of apnea and hypopnea events per hour. Subjects with $\mathrm{AHI}<15$ were classified as simple snorers, subjects with $\mathrm{AHI} \geq 15$ and $<30$ were classified as having mild to moderate $\mathrm{SDB}$, and subjects with $\mathrm{AHI} \geq 30$ were classified as severe SDB patients. ${ }^{3,27}$ The change in AHI was calculated as the follow-up AHI-baseline AHI. The supine time $\%$ was calculated as [total supine time $(\mathrm{min}) /$ total sleep time $(\mathrm{min})] \times 100$. The change in supine time $\%$ was calculated as supine time $\%$ of the follow up-supine time $\%$ of the baseline.

\section{Statistical analysis}

The subjects' characteristics were expressed as mean \pm standard deviation (SD) for continuous variables, and as frequency or percentage for categorical variables. Differences in characteristics between the baseline and follow-up evaluations were assessed by paired t-test. One-way analysis of variance was used to compare characteristics between subgroups. Pearson's correlation analysis was performed to assess the association between the change in AHI and other variables. In order to evaluate which factor affects the change in AHI, multiple linear regression analysis was performed. Two-tailed p-values of less than 0.05 were considered to be statistically significant. All statistical analyses were conducted using the statistical software package, SPSS for Windows, version 22 (SPSS, Chicago, IL, USA).

\section{RESULTS}

A total of 56 middle aged to elderly subjects at the baseline (61.2 \pm 5.7 years) were included in this study, and $84 \%$ of them were male. The baseline demographic characteristics and polysomnographic data are summarized in Table 1. The mean AHI and BMI were $30.3 \pm 16.9$ and $24.8 \pm 2.5$. After a mean interval of $62.4 \pm 22.0$ months (range from 26 to 110 ) between the baseline and follow-up PSG, we found no significant changes in $\mathrm{AHI}$, supine $\mathrm{AHI}$, oxygen desaturation index (ODI), BMI or minimal $\mathrm{O}_{2}$ saturation. Supine time $\%(60.2 \pm 28.1$ vs. $53.5 \pm 29.4)$ and ESS score $(9.3 \pm 4.3$ vs. $8.1 \pm 4.8)$ were significantly decreased at the follow-up evaluation. Thirteen subjects were simple snorers at baseline, but five of them were changed to 
mild to moderate SDB at follow-up. Among the 15 mild to moderate SDB subjects at baseline, 4 were changed to simple snorers and 5 were changed to the severe SDB group. In the severe SDB group at baseline, 3 were changed to simple snorers, 10 were changed to the mild to moderate SDB group and 16 subjects remained in the severe SDB group.

In the subgroup analysis based on the severity of SDB in baseline, there were no difference in baseline age, proportion of male gender, weight, BMI, supine time \%, ESS score, or interval between baseline and follow-up evaluation among the 3 groups (Table 2). The AHI of the severe SDB group decreased at the follow-up evaluation $(43.9 \pm 10.6$ vs. $35.6 \pm 20.0, \mathrm{p}=0.009)$ (Figure 1) in the intra-group analysis, and the simple snorer group showed a tendency to deteriorate $(\mathrm{p}=0.079)$. However, supine AHI was not changed in any of the 3 groups.

The correlation analysis showed that the change in supine time $\%(\mathrm{R}=0.424, \mathrm{p}=0.001)$ and the baseline $\mathrm{AHI}(\mathrm{R}=-0.323$, $\mathrm{p}=0.015$ ) (Figure 2 ) were significantly correlated with the change in $\mathrm{AHI}$, but the follow-up duration $(\mathrm{R}=0.016, \mathrm{p}=0.904)$ or base- line $\mathrm{BMI}(\mathrm{R}=0.08, \mathrm{p}=0.557)$ was not significantly correlated with changes in AHI. In addition, there was no significant association between the follow-up duration and the change in supine time $\%(\mathrm{R}=-0.108, \mathrm{p}=0.429)$. The result of the multiple regression analysis for the change in $\mathrm{AHI}$ is shown in Table 3. In the regression analysis, the change in $\mathrm{AHI}$ was found to be associated with the change in supine time $\%(\beta=0.387, \mathrm{p}=0.003)$ and the baseline AHI $(\beta=-0.272, p=0.037)$. The association with the change in BMI, baseline age, sex and the change in percentage of REM sleep were insignificant.

\section{DISCUSSION}

In the present study, 56 middle-aged and elderly SDB patients were followed-up over an average of 5.1 years, and we found that untreated SDB as a whole did not progress over time. However, the severe SDB group did improve and the mild SDB group showed a tendency to become aggravated over time in our subgroup analysis. The change in supine time $\%$

Table 1. Comparison of demographic and polysomnographic data between baseline and follow up

\begin{tabular}{|c|c|c|c|}
\hline & Baseline (mean $\pm \mathrm{SD})$ & Follow up (mean \pm SD) & p-value \\
\hline Age (yr) & $61.2 \pm 5.71$ & $66.0 \pm 5.63$ & $<0.001$ \\
\hline Sex (male/female) & $47 / 9(84 \% / 16 \%)$ & & \\
\hline Follow up interval (mo) & & $62.4 \pm 22.0$ & \\
\hline BMI $\left(\mathrm{kg} / \mathrm{m}^{2}\right)$ & $24.8 \pm 2.5$ & $25.2 \pm 3.4$ & 0.093 \\
\hline ESS score & $9.3 \pm 4.3$ & $8.1 \pm 4.8$ & 0.037 \\
\hline AHI (events/h) & $30.3 \pm 16.9$ & $27.0 \pm 18.2$ & 0.097 \\
\hline Severity & & & 0.092 \\
\hline Simple snorer & 13 & 15 & \\
\hline Mild to moderate & 15 & 21 & \\
\hline Severe & 28 & 20 & \\
\hline Supine AHI (events/h) & $43.6 \pm 25.2$ & $43.7 \pm 23.4$ & 0.977 \\
\hline ODI (events/h) & $22.7 \pm 15.8$ & $21.5 \pm 18.3$ & 0.444 \\
\hline Snoring (\%) & $31.3 \pm 21.3$ & $25.1 \pm 20.0$ & 0.039 \\
\hline Supine time (\%) & $60.2 \pm 28.1$ & $53.5 \pm 29.4$ & 0.048 \\
\hline Minimal SpO2 (\%) & $79.1 \pm 12.8$ & $82.0 \pm 8.6$ & 0.108 \\
\hline Sleep period time & $442.2 \pm 46.8$ & $449.2 \pm 37.6$ & 0.366 \\
\hline Total sleep time & $367.1 \pm 47.9$ & $375.7 \pm 45.7$ & 0.337 \\
\hline Sleep efficiency & $81.3 \pm 62.1$ & $80.4 \pm 49.2$ & 0.604 \\
\hline Sleep latency & $10.5 \pm 11.5$ & $13.5 \pm 9.0$ & 0.298 \\
\hline WASO & $75.2 \pm 15.4$ & $78.2 \pm 16.7$ & 0.684 \\
\hline S1 (\%) & $16.0 \pm 8.6$ & $13.9 \pm 7.8$ & 0.086 \\
\hline S2 (\%) & $51.1 \pm 11.3$ & $48.6 \pm 10.3$ & 0.198 \\
\hline S3 (\%) & $2.2 \pm 4.1$ & $4.6 \pm 6.4$ & 0.007 \\
\hline REM (\%) & $14.2 \pm 5.7$ & $16.6 \pm 7.2$ & 0.027 \\
\hline
\end{tabular}

Data are presented as mean \pm SD or frequency. BMI: body mass index, ESS: Epworth sleepiness scale, AHI: apnea-hypopnea index, ODI: oxygen desaturation index, WASO: wake after sleep onset, S1: stage 1 sleep, S2: stage 2 sleep, S3: stage 3 sleep, REM: rapid eye movement sleep 
and the baseline AHI were significantly associated with the change in AHI, but a minimal weight change did not predict SDB progression.

In contrast to the present study, many previous studies showed that SDB had a tendency to worsen over time. Large population based studies such as the Wisconsin Sleep Cohort, ${ }^{11}$ the Sleep Heart Health Study, ${ }^{8}$ and the Cleveland Family study ${ }^{9}$ have demonstrated that SDB progressed over time. One other study, with 160 clinical subjects, also reported the deteriorative course of SDB. ${ }^{12}$ The progression of SDB seen in these studies was greatly related to weight gain, and they all observed that weight gain played a critical role for longitudinal changes in the severity of SDB. Even studies that negated progressive deterioration of untreated SDB admitted that weight change affected the natural course of SDB. ${ }^{16,17}$ Given the known relationship between body weight and severity of SDB from cross sectional studies, ${ }^{27-29}$ it seems plausible that weight gain could exacerbate SDB over time. We found no significant association between weight change and the course of SDB, and it might be due to minimal weight changes displayed by our subjects $(-0.19 \pm$ $2.69 \mathrm{~kg}$; ranged -6.0 to $8.8 \mathrm{~kg}$ ) during the observational period. On the other hand, several other studies have reported an in-

Table 2. Changes in clinical and polysomnographic parameters at baseline and follow up in 3 SDB severity groups

\begin{tabular}{|c|c|c|c|}
\hline & Simple snorers, AHI $<15(\mathrm{~N}=13)$ & Mild to moderate $\mathrm{SDB}, 15 \leq \mathrm{AHI}<30(\mathrm{~N}=15)$ & Severe $\mathrm{SDB}, \mathrm{AHI} \geq 30(\mathrm{~N}=28)$ \\
\hline Male, N (\%) & $13(100)$ & $11(73.3)$ & $23(82.1)$ \\
\hline Baseline age (yr) & $60.4 \pm 4.6$ & $61.1 \pm 4.6$ & $61.6 \pm 6.8$ \\
\hline Baseline weight (kg) & $70.3 \pm 11.4$ & $67.1 \pm 10.0$ & $68.6 \pm 8.7$ \\
\hline Interval (mo.) & $62.2 \pm 22.0$ & $60.6 \pm 21.1$ & $63.4 \pm 23.1$ \\
\hline \multicolumn{4}{|l|}{$\mathrm{BMI}(\mathrm{kg} / \mathrm{m})$} \\
\hline Baseline & $24.8 \pm 2.7$ & $24.0 \pm 2.3$ & $25.3 \pm 2.4$ \\
\hline Follow-up & $26.1 \pm 5.1$ & $24.0 \pm 2.6$ & $25.5 \pm 2.7$ \\
\hline p-value & 0.177 & 0.893 & 0.300 \\
\hline \multicolumn{4}{|l|}{ AHI (event/h) } \\
\hline Baseline & $7.6 \pm 3.5$ & $24.4 \pm 3.1$ & $43.9 \pm 10.6$ \\
\hline Follow-up & $12.9 \pm 9.8$ & $23.4 \pm 10.6$ & $35.6 \pm 20.0$ \\
\hline $\mathrm{p}$-value & 0.074 & 0.719 & 0.009 \\
\hline \multicolumn{4}{|l|}{ Supine AHI (event/h) } \\
\hline Baseline & $13.6 \pm 9.1$ & $34.7 \pm 14.8$ & $62.3 \pm 17.5$ \\
\hline Follow-up & $57.3 \pm 24.5$ & $57.3 \pm 23.4$ & $49.7 \pm 34.5$ \\
\hline $\mathrm{p}$-value & 0.079 & 0.413 & 0.139 \\
\hline \multicolumn{4}{|l|}{ Supine time (\%) } \\
\hline Baseline & $56.7 \pm 30.1$ & $72.6 \pm 23.8$ & $55.2 \pm 28.3$ \\
\hline Follow-up & $57.3 \pm 24.5$ & $57.3 \pm 23.4$ & $49.7 \pm 34.5$ \\
\hline $\mathrm{p}$-value & 0.929 & 0.004 & 0.306 \\
\hline \multicolumn{4}{|l|}{ ODI (event/h) } \\
\hline Baseline & $4.4 \pm 2.7$ & $17.9 \pm 6.0$ & $33.7 \pm 13.7$ \\
\hline Follow-up & $7.6 \pm 6.7$ & $17.4 \pm 9.6$ & $30.1 \pm 20.8$ \\
\hline p-value & 0.080 & 0.844 & 0.193 \\
\hline \multicolumn{4}{|l|}{ REM (\%) } \\
\hline Baseline & $17.5 \pm 6.3$ & $14.4 \pm 6.1$ & $12.6 \pm 4.6$ \\
\hline Follow-up & $14.8 \pm 8.1$ & $19.0 \pm 6.8$ & $16.1 \pm 6.9$ \\
\hline p-value & 0.186 & 0.037 & 0.019 \\
\hline \multicolumn{4}{|l|}{ ESS } \\
\hline Baseline & $10.3 \pm 4.4$ & $7.3 \pm 5.4$ & $9.9 \pm 3.4$ \\
\hline Follow-up & $10.1 \pm 4.9$ & $5.5 \pm 3.4$ & $8.5 \pm 4.9$ \\
\hline $\mathrm{p}$-value & 0.869 & 0.043 & 0.125 \\
\hline
\end{tabular}

Data are presented as mean \pm SD. SDB: sleep-disordered breathing, BMI: body mass index, AHI: apnea-hypopnea Index, ODI: oxygen desaturation index, REM: rapid eye movement sleep, ESS: Epworth sleepiness scale 
significant relationship between weight change and SDB progression. ${ }^{13,15,18,30}$ Several factors, other than fat deposition due to weight gain, have been suggested as a cause for the aggra-

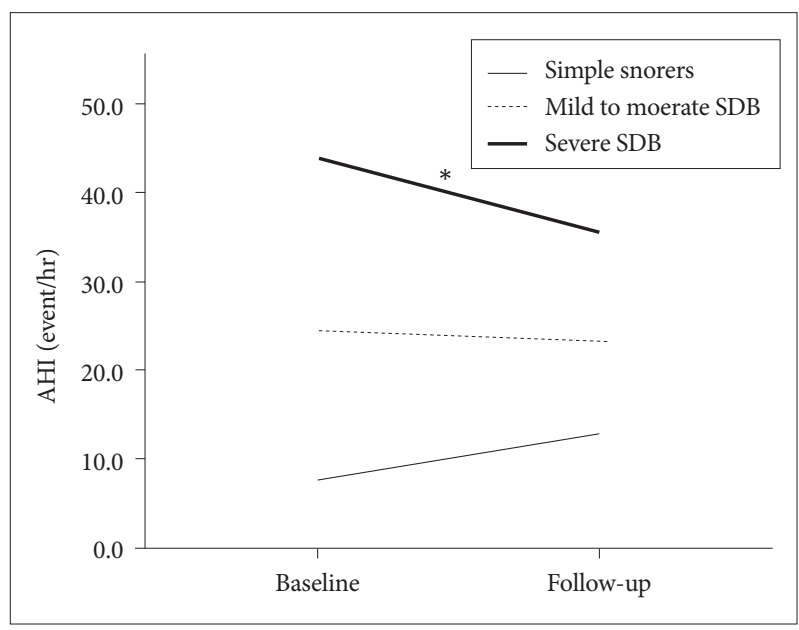

Figure 1. Changes in apnea-hypopnea index (AHI) between baseline and follow-up in 56 untreated sleep disordered breathing (SDB) patients. The AHI of severe SDB group significantly improved over time $(p=0.009) .{ }^{*} p$-values less than 0.05 .

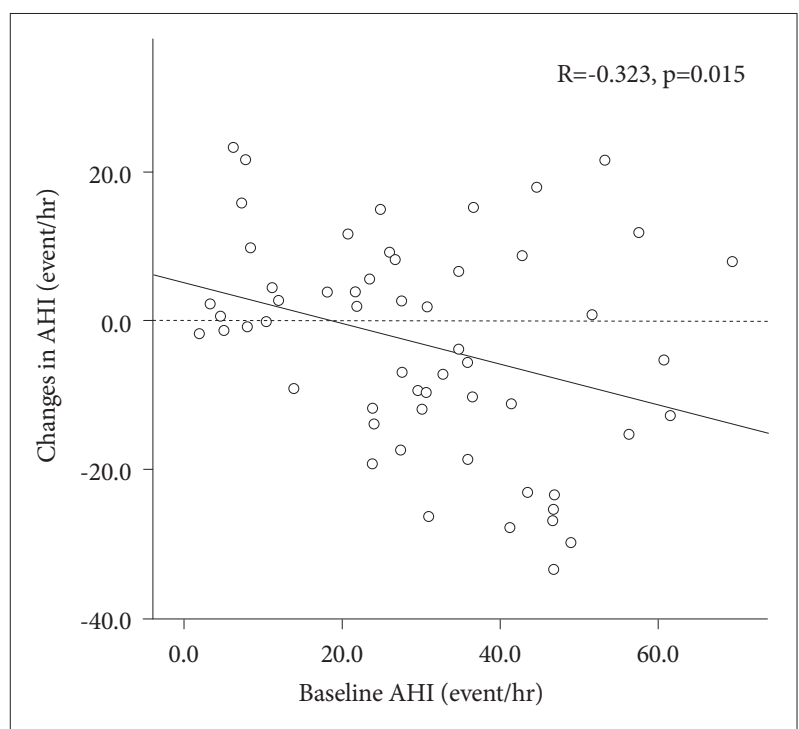

Figure 2. Correlation between the baseline apnea-hypopnea in$\operatorname{dex}(\mathrm{AHI})$ and the change in $\mathrm{AHI}$. vation of SDB. These include local mechanical trauma, ${ }^{30}$ pharyngeal nerve damage, ${ }^{31}$ progressive loss of airway reflex, ${ }^{32}$ pharyngeal muscle lesions ${ }^{33}$ and ventilatory instability, ${ }^{34}$ which all are considered to occur as a result of snoring.

In the present study, the severe SDB group improved significantly, and there was a trend of aggravation in simple snorers. This result is consistent with the majority of previous studies. Sforza et al. ${ }^{19}$ clearly stated that they saw a negative correlation between the baseline respiratory disturbance index (RDI) and the change in RDI, and Fisher et al. ${ }^{17}$ also reported that the improved group had significantly higher RDI at the baseline observation. It is also important to note that most studies supporting a natural worsening course of SDB studied subjects with a mild syndrome of disease at the baseline (AHI: 2.6-9.0). Considering the result of the present study and those of previous studies, it seems that mild SDB tends to worsen and severe SDB tends to improve over time. The mechanism of the progression and regression of SDB, according to baseline severity, remains uncertain. Anatomical and functional change of the upper airway, induced by chronic repetitive obstruction, could be part of the mechanism of deterioration of mild SDB patients. ${ }^{35}$ If the increase in AHI reaches a critical point, the demand of the body to protect sleep from intolerable hypoxemia may prevent further exacerbation and preserve optimal ventilation. ${ }^{36}$

There is a possibility that improvement shown in the severe SDB patients in the present study was affected by the positional change between polysomnographic examinations. Supine time \% was significantly decreased in the mild to moderate SDB group with the severe SDB group showing a weak tendency to decrease. It has yet to be determined that SDB patients show the change in sleeping position over time. Meanwhile, sleep position has been previously shown to either aggravate, or improve SDB. Lying in the supine position can increase the level of respiratory distress by 40 to $50 \%,,^{37,38} \mathrm{com}$ pared to lying in a lateral position due to the effect of gravity which narrows the pharyngeal cross-sectional area. ${ }^{39,40}$ However, in the regression analysis of the present study, the baseline SDB severity was negatively associated with changes in

Table 3. Regression analysis of $\mathrm{AHI}$ changes

\begin{tabular}{lccc}
\hline & Unstandardized B & Standardized $\beta$ & p-value \\
\hline Changes in supine time (\%) & 0.224 & 0.387 & 0.003 \\
Baseline AHI (event/hr) & -0.230 & -0.272 & 0.037 \\
Changes in BMI (kg/m²) & 1.055 & 0.133 & 0.289 \\
Age at baseline (yr) & -0.131 & -0.052 & 0.672 \\
Changes in REM (\%) & -0.062 & -0.034 & 0.794 \\
Sex & 0.335 & 0.009 & 0.943 \\
\hline
\end{tabular}

AHI: apnea-hypopnea index, BMI: body mass index, REM: rapid eye movement sleep 
SDB severity after controlling for the positional change.

There are several limitations to the current study. First, the present study included patients who visited a sleep clinic, and this might lead to selection bias reflected by a high proportion of severe and male cases. However, the result of this study could be still clinically meaningful as complications of SDB develop frequently and seriously in severe SDB. Second, the present study has a retrospective design, which limits the interpretation of this study in terms of the natural course of the illness. Third, changes in weight of the subjects were minimal to evaluate the effect of weight change on the long-term change of SDB.

In summary, untreated middle aged and elderly SDB patients did not deteriorate over a mean period of 5 years with modest improvement in severe SDB. The clinical course of SDB was associated with changes in supine time percent and the baseline SDB severity. There is a possibility that a proportion of severe SDB patients can expect improvement in SDB severity irrespective of changes in sleep position or body weight.

\section{Acknowledgments}

This study was supported by the Basic Science Research Program through the National Research Foundation of Korea (NRF) funded by the Ministry of Education, Science and Technology (Grant No. 2010-0008886).

\section{REFERENCES}

1. Young T, Palta M, Dempsey J, Skatrud J, Weber S, Badr S. The occurrence of sleep-disordered breathing among middle-aged adults. $\mathrm{N}$ Engl J Med 1993;328:1230-1235.

2. Ancoli-Israel S, Kripke DF, Klauber MR, Mason WJ, Fell R, Kaplan O. Sleep-disordered breathing in community-dwelling elderly. Sleep 1991; 14:486-495.

3. Lee SD, Kang SH, Ju G, Han JW, Kim TH, Lee CS, et al. The prevalence of and risk factors for sleep-disordered breathing in an elderly Korean population. Respiration 2014;87:372-378.

4. Shahar E, Whitney CW, Redline S, Lee ET, Newman AB, Nieto FJ, et al. Sleep-disordered breathing and cardiovascular disease: cross-sectional results of the Sleep Heart Health Study. Am J Respir Crit Care Med 2001; 163:19-25.

5. Coughlin SR, Mawdsley L, Mugarza JA, Calverley PM, Wilding JP. Obstructive sleep apnoea is independently associated with an increased prevalence of metabolic syndrome. Eur Heart J 2004;25:735-741.

6. Peppard PE, Young T, Palta M, Skatrud J. Prospective study of the association between sleep-disordered breathing and hypertension. N Engl J Med 2000;342:1378-1384.

7. Uyrum E, Balbay O, Annakkaya AN, Gulec Balbay E, Silan F, Arbak P. The relationship between obstructive sleep apnea syndrome and apolipoprotein E genetic variants. Respiration 2015;89:195-200.

8. Newman AB, Foster G, Givelber R, Nieto FJ, Redline S, Young T. Progression and regression of sleep-disordered breathing with changes in weight: the Sleep Heart Health Study. Arch Intern Med 2005;165:24082413.

9. Redline S, Schluchter MD, Larkin EK, Tishler PV. Predictors of longitudinal change in sleep-disordered breathing in a nonclinic population. Sleep 2003;26:703-709.

10. Young T, Peppard PE, Gottlieb DJ. Epidemiology of obstructive sleep apnea: a population health perspective. Am J Respir Crit Care Med 2002; 165:1217-1239.

11. Peppard PE, Young T, Palta M, Dempsey J, Skatrud J. Longitudinal study of moderate weight change and sleep-disordered breathing. JAMA 2000; 284:3015-3021.

12. Berger G, Berger R, Oksenberg A. Progression of snoring and obstructive sleep apnoea: the role of increasing weight and time. Eur Respir J 2009;33:338-345.

13. Sahlman J, Pukkila M, Seppa J, Tuomilehto H. Evolution of mild obstructive sleep apnea after different treatments. Laryngoscope 2007;117:11071111.

14. Inoue Y, Nanba K, Hazama G, Takata K, Arai H. Long-term follow-up study on patients with sleep apnea syndrome. Psychiatry Clin Neurosci 2001;55:245-246.

15. Sforza E, Addati G, Cirignotta F, Lugaresi E. Natural evolution of sleep apnoea syndrome: a five year longitudinal study. Eur Respir J 1994;7:17651770 .

16. Ancoli-Israel S, Gehrman P, Kripke DF, Stepnowsky C, Mason W, Cohen-Zion $\mathrm{M}$, et al. Long-term follow-up of sleep disordered breathing in older adults. Sleep Med 2001;2:511-516.

17. Fisher D, Pillar G, Malhotra A, Peled N, Lavie P. Long-term follow-up of untreated patients with sleep apnoea syndrome. Respir Med 2002;96: 337-343.

18. Bliwise DL. Epidemiology of Age-Dependence in Sleep Disordered Breathing (Sdb) in Old Age: The Bay Area Sleep Cohort (Basc). Sleep Med Clin 2009;4:57-64.

19. Sforza E, Gauthier M, Crawford-Achour E, Pichot V, Maudoux D, Barthelemy JC, et al. A 3-year longitudinal study of sleep disordered breathing in the elderly. Eur Respir J 2012;40:665-672.

20. Svanborg E, Larsson H. Development of nocturnal respiratory disturbance in untreated patients with obstructive sleep apnea syndrome. Chest 1993;104:340-343.

21. Oksenberg A, Silverberg DS, Arons E, Radwan H. Positional vs nonpositional obstructive sleep apnea patients: anthropomorphic, nocturnal polysomnographic, and multiple sleep latency test data. Chest 1997;112: 629-639.

22. Cao J, Que C, Wang G, He B. Effect of posture on airway resistance in obstructive sleep apnea-hypopnea syndrome by means of impulse oscillation. Respiration 2009;77:38-43.

23. Kim T, Lee CS, Lee SD, Kang SH, Han JW, Malhotra A, et al. Impacts of comorbidities on the association between arterial stiffness and obstructive sleep apnea in the elderly. Respiration 2015;89:304-311.

24. Lee SD, Ju G, Choi JA, Kim JW, Yoon IY. The association of oxidative stress with central obesity in obstructive sleep apnea. Sleep Breath 2012; 16:511-517.

25. Kales A, Rechtschaffen A. A Manual of Standardized Terminology, Techniques and Scoring System for Sleep Stages of Human Subjects. Washington: US Department of Health, Education and Welfare, Public Health Service, National Institutes of Health, National Institute of Neurological Diseases and Blindness, Neurological Information Network;1968.

26. Quan S, Gillin JC, Littner M, Shepard J. Sleep-related breathing disorders in adults: recommendations for syndrome definition and measurement techniques in clinical research. editorials. Sleep 1999;22:662689.

27. Young T, Shahar E, Nieto FJ, Redline S, Newman AB, Gottlieb DJ, et al. Predictors of sleep-disordered breathing in community-dwelling adults: the Sleep Heart Health Study. Arch Intern Med 2002;162:893-900.

28. Newman AB, Nieto FJ, Guidry U, Lind BK, Redline S, Pickering TG, et al. Relation of sleep-disordered breathing to cardiovascular disease risk factors: the Sleep Heart Health Study. Am J Epidemiol 2001;154:50-59.

29. Araz O, Yilmazel Ucar E, Dorman E, Bayraktutan Z, Yayla M, Yilmaz $\mathrm{N}$, et al. Is there a relationship between obstructive sleep apnea syndrome severity and nesfatin-1? Respiration 2015;90:105-110.

30. Lindberg E, Elmasry A, Gislason T, Janson C, Bengtsson H, Hetta J, et al. Evolution of sleep apnea syndrome in sleepy snorers: a populationbased prospective study. Am J Respir Crit Care Med 1999;159:2024-2027.

31. Pendlebury ST, Pepin JL, Veale D, Levy P. Natural evolution of moderate 
sleep apnoea syndrome: significant progression over a mean of 17 months. Thorax 1997;52:872-878.

32. Horner RL, Innes JA, Murphy K, Guz A. Evidence for reflex upper airway dilator muscle activation by sudden negative airway pressure in man. J Physiol 1991;436:15-29.

33. Edström L, Larsson H, Larsson L. Neurogenic effects on the palatopharyngeal muscle in patients with obstructive sleep apnoea: a muscle biopsy study. J Neurol Neurosurg Psychiatry 1992;55:916-920.

34. Onal E, Lopata M. Periodic breathing and the pathogenesis of occlusive sleep apneas. Am Rev Respir Dis 1982;126:676-680.

35. Fleetham JA. Upper airway imaging in relation to obstructive sleep apnea. Clin Chest Med 1992;13:399-416.

36. Grunstein RR. Sleep apnoea--evolution and doubt. Eur Respir J 1994;7:
1741-1743.

37. Cartwright RD. Effect of sleep position on sleep apnea severity. Sleep 1984;7:110-114.

38. Phillips BA, Okeson J, Paesani D, Gilmore R. Effect of sleep position on sleep apnea and parafunctional activity. Chest 1986;90:424-429.

39. Brown IB, McClean PA, Boucher R, Zamel N, Hoffstein V. Changes in pharyngeal cross-sectional area with posture and application of continuous positive airway pressure in patients with obstructive sleep apnea. Am Rev Respir Dis 1987;136:628-632.

40. Yildirim N, Fitzpatrick M, Whyte K, Jalleh R, Wightman A, Douglas $\mathrm{N}$. The effect of posture on upper airway dimensions in normal subjects and in patients with the sleep apnea/hypopnea syndrome. Am Rev Respir Dis 1991;144:845-847. 